
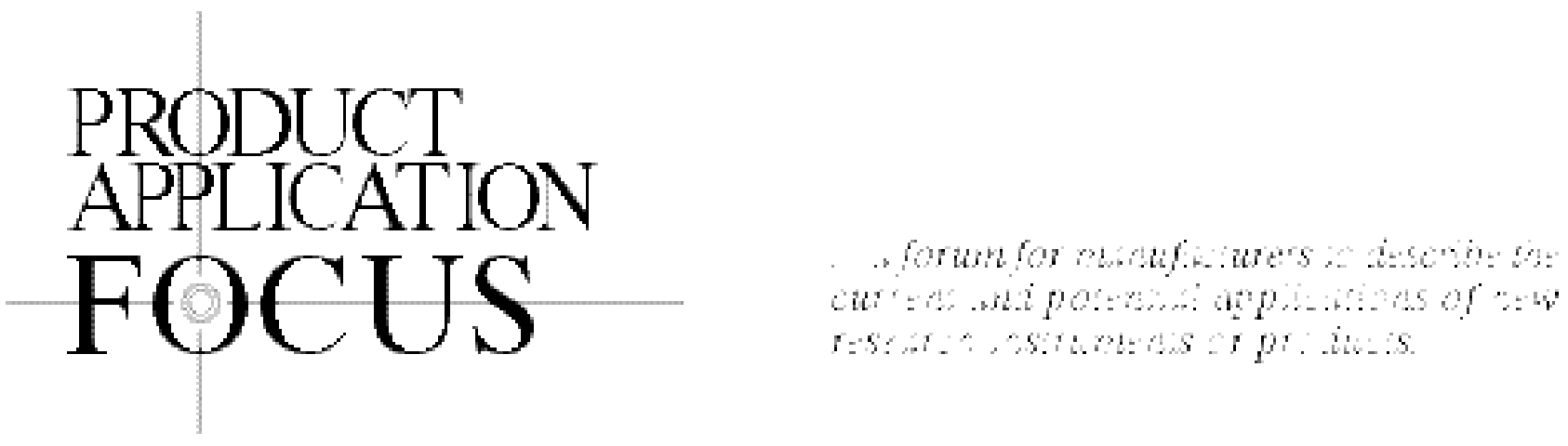

\title{
High-Quality RNA from Cells Isolated by Laser Capture Microdissection
}

\author{
A. Mikulowska-Mennis, T.B. Taylor, P. Vishnu, S.A. Michie ${ }^{1}$, R. Raja, N. Horner, and \\ S.T. Kunitake
}

Arcturus, Mountain View, CA, and ${ }^{1}$ Stanford University School of Medicine, Stanford, CA, USA

BioTechniques 33:176-179(July 2002)

\section{ABSTRACT}

Laser capture microdissection (LCM) provides a rapid and sim ple method for procuring homogeneous populations of cells. However, reproducible isolation of intact RNA from these cells can be problematic; the sample may deteriorate before or during sectioning, RNA may degrade during slide staining and LCM, and inadequate extraction and isolation methods may lead to poor recovery.

Our report describes an optimized protocol for preparation of frozen sections for LCM using the HistoGene ${ }^{\mathrm{TM}}$ Frozen Section Staining Kit. This slide preparation method is combined with the PicoPure $^{\mathrm{TM}}$ RNA Isolation Kit for extraction and isolation of RNA from low numbers of microdissected cells. The procedure is easy to perform, rapid, and reproducible. Our results show that the RNA isolated from the LCM samples prepared according to our protocol is of high quality. The RNA maintains its integrity as shown by RT-PCR detection of genes of different abundance levels and by electrophoretic analysis of ribosomal RNA. RNA obtained by this method has also been used to synthesize probes for interrogating cDNA microarray analyses to study expression levels of thousands of genes from LCM samples.

\section{INTRODUCTION}

The elucidation of the human genome and the development of sensitive and powerful analytical tools have given scientists the unprecedented opportunity to study intracellular mechanisms on a molecular level. To achieve the greatest level of understanding, the molecular analysis should be performed on pure cell populations taken from their native environment $(11,15)$. Since a tissue biopsy consists of a heterogeneous population of cells and extracellular material, the analysis of such material may yield misleading or confusing molecular results. Cells in culture can be homogenous but do not necessarily reflect the in vivo conditions. Therefore, the need is great for a method to obtain pure cell subpopulations from tissue or biopsy samples to yield accurate information from molecular analysis.

Laser capture microdissection (LCM), conceived by scientists at the National Cancer Institute, provides a rapid and simple method for procuring homogeneous populations of cells for analysis $(2,5,16)$. Frozen sections of tissue are cut, fixed, stained, and dehydrated before LCM. Once placed on 
the PixCell ${ }^{\mathrm{TM}}$ stage (Arcturus, Mountain View, CA, USA), the entire non-coverslipped sample can be visualized through the microscope ocular or on the video monitor to locate the cell(s) of interest. LCM employs positive selection to procure cells from tissue sections. CapSure ${ }^{\mathrm{TM}}$ Transfer Film (Arcturus), a transparent thermoplastic film attached to a proprietary optical-grade plastic cap, is positioned over the cells, and a lowpower infrared laser pulse, with beam sizes of 7.5-30 $\mu \mathrm{m}$, is triggered to capture a cell or multiple cells. The laser pulse activates the transfer film, and the cells within the laser beam area adhere to the film. At no time does the laser directly impact the tissue sample. Other cells within the area under the cap can then be identified and targeted; once all selected cells have been captured, the transfer film cap with the cells attached to the film surface is lifted. Thus, the desired cells are microdissected while the surrounding tissue remains intact on the slide. The transfer film cap can be placed directly into a microcentrifuge tube, and the cell contents can be extracted for molecular studies to evaluate genetic alterations, cellular protein contents, and cell-specific gene expression $(1,3,6-8$, 10-12,15,17-19 and http://www.ncbi.nlm.nih.gov/ncicgap).

RNA has been recovered successfully from LCM samples and used in a number of analyses $(4,9,11,14,15)$. However, reproducible isolation of intact RNA can be problematic; the sample may deteriorate before or during sectioning, RNA may degrade during slide staining and LCM, and inadequate extraction and isolation methods can lead to poor recovery. Here we describe an optimized process for the isolation of high-quality RNA from LCM samples. We will highlight procedures for sample handling, slide staining, and sample extraction.

\section{MATERIALS A ND METHODS}

\section{Animals, Tissue Harvesting, and Cryo-Preservation}

Salivary glands were obtained from three, 20-week-old male non-obese diabetic (NOD) mice. Thymus, brain, small intestine, liver, and kidney were obtained from five BALB/c 10-30-weeks-old female mice. Mice were euthanized, and the tissues were rapidly removed under RNase-free conditions. The tissues were frozen in OCT (Tissue Tek; Sakura Finetek USA,Torrance, CA, USA) on dry ice immediately upon dissection. The blocks were stored at $-86^{\circ} \mathrm{C}$.

\section{Slide Preparation and LCM}

Before sectioning, the cryostat (Leica CM 3050, Leica Microsystems, Deerfield, IL, USA) was wiped down with $100 \%$ ethanol to avoid cross contamination, and a fresh disposable blade was used to cut each tissue. The tissue was placed in the cryostat for about $10 \mathrm{~min}$ to adjust to the cutting temperature $\left(-15^{\circ} \mathrm{C}-20^{\circ} \mathrm{C}\right)$. Sections $(8 \mu \mathrm{m}$ thick) were cut and placed on room-temperature Silane Prep slides (Sigma, St. Louis, MO, USA); the slides were stored in a slide box on dry ice until the cutting was completed. Unless immediately processed, the slides were then stored at $-70^{\circ} \mathrm{C}$. A HistoGene ${ }^{\mathrm{TM}}$ staining kit (Arcturus) was used to prepare the tissues for LCM. Briefly, the slides were thawed for $30 \mathrm{~s}$, fixed in nuclease-free $75 \%$ ethanol, rehydrated in nuclease-free distilled water for $30 \mathrm{~s}$, stained with HistoGene stain for $20 \mathrm{~s}$, and rinsed in nuclease-free water for $30 \mathrm{~s}$. They were then dehydrated by sequential immersion into $75 \%, 95 \%$, and $100 \%$ ethanol for $30 \mathrm{~s}$ each, then cleared in xylene for $5 \mathrm{~min}$, and dried for $5 \mathrm{~min}$. Disposable staining jars were filled with fresh solutions for each batch of slides.

Staining was followed by immediate LCM performed on a Pixcell II ${ }^{\circledR}$ LCM Instrument. All microdissections were done in triplicates using CapSure LCM caps. Five hundred cells were collected using the $7.5 \mu \mathrm{m}$ diameter laser spot size. Additional caps with 10 Purkinje cells/cap were collected for some experiments.

\section{RNA Isolation and RT-PCR}

Microdissected cells were immediately processed using the PicoPure ${ }^{\mathrm{TM}}$ RNA isolation kit (Arcturus). This kit, which uses guandinium thiocyanate lysis followed by a spin column purification method, has been optimized for the solubilization and recovery of RNA from LCM samples. Briefly, $20 \mu \mathrm{L}$ extraction buffer were placed into the LCM cap/microcentrifuge tube assembly and incubated for $30 \mathrm{~min}$ at $42^{\circ} \mathrm{C}$. After incubation, the assembly was briefly centrifuged to collect the extraction fluid in the microcentrifuge tube. The fluid was then loaded onto a spin column, washed several times, and the total cellular RNA was eluted in a $10-\mu \mathrm{L}$ volume.

For RT-PCR analysis, $10 \mu \mathrm{L}$ isolated RNA were mixed with $10 \mu \mathrm{L}$ reverse transcriptase master mixture for the synthesis of cDNA (Sensiscript Reverse Transcriptase Handbook; Qiagen, Valencia, CA, USA) using oligo-dT primer (diluted 1:10 in RNase-free water) and RNase inhibitor (diluted 1:4 in $1 \times$ reverse transcriptase buffer) (Invitrogen, Carlsbad, CA, USA). The reverse transcription reaction was carried out for $1 \mathrm{~h}$ at $37^{\circ} \mathrm{C}$, followed by a 5 -min denaturation step at $95^{\circ} \mathrm{C}$ as described by the manufacturer.

PCR was then performed using the cDNA and primers for mouse glyceraldehyde-3-phosphate-dehydrogenase (GAPDH) (forward, 5'-ACCACAGTCCATGCCATCAC-3'; reverse, 5' TCCACCACCCTGTTGCTGTA- $3^{\prime}$ ), mouse $\beta$-actin (forward, 5'-CGTGGGCCGCCCTAGGCACCA-3'; reverse: 5'-TTG GCCTTAGGGTTCAGGGGGG-3') or for three different abundance level genes as provided in the Control RT-PCR Primer Kit (high abundance, elongation factor-1; medium abundance, GAPDH; low abundance, protein phosphatase 1; Stratagene, La Jolla, CA, USA). Using a $0.5 \mathrm{~mL}$ thin-walled tube, $2 \mu \mathrm{L}$ synthesized cDNA were added to $23 \mu \mathrm{L}$ master mixture containing forward and reverse primers (final concentration of $200 \mathrm{nM}$ each), along with one Ready-to-Go Bead (Amersham Biosciences, Piscataway, NJ, USA). Samples were then denatured at $95^{\circ} \mathrm{C}$ for $5 \mathrm{~min}$ and amplified for 35 cycles of $95^{\circ} \mathrm{C}$ for $15 \mathrm{~s}$, primer-specific annealing temperature for $30 \mathrm{~s}$, and $72^{\circ} \mathrm{C}$ for $1 \mathrm{~min}$, with a final extension at $72^{\circ} \mathrm{C}$ for $10 \mathrm{~min}$.

Gel loading buffer was added to the PCR sample, and 20 $\mu \mathrm{L}$ of this mixture were separated on a $6 \%$ Novex acrylamide gel (Invitrogen). The gel was stained with SYBR Gold ${ }^{\circledR} \mathrm{Nu}-$ cleic Acid Gel Stain (Molecular Probes, Eugene, OR, USA) and imaged on a fluorimager (Molecular Dynamics, Sunnyvale, CA, USA). 


\section{Microchannel Electrophoresis}

RNA was isolated as described above from $(i)$ an intact (not stained or dehydrated) frozen section of mouse liver, (ii) 15000 cells captured from a section prepared according to the HistoGene protocol, and (iii) 15000 cells captured from a section that was dehydrated but not stained. The RNAs were electrophoretically analyzed (13), and the gross quality of $18 \mathrm{~S}$ and $28 \mathrm{~S}$ ribosomal RNAs was assessed using a Bioanalyzer 2000 and the RNA 6000 LabChip ${ }^{\circledR}$ kit (Agilent, Palo Alto, CA, USA).

\section{cDNA Microarray Hybridization}

Isolated mouse RNA was used to prepare probes to study gene expression using cDNA microarrays. Briefly, isolated RNA was amplified using the RiboAmp ${ }^{\mathrm{TM}}$ RNA linear amplification kit (Arcturus). This process linearly amplifies mRNA, creating antisense RNA (aRNA). Three micrograms of aRNA were converted to Cy5-labeled cDNA. The probe was hybridized in a hybridization solution containing $50 \%$ formamide to an 8880-element mouse cDNA microarray for 14 $\mathrm{h}$ at $42^{\circ} \mathrm{C}$. After washing, the array was scanned on a GenePix 4000A scanner (Axon Instruments, Union City, CA, USA).

\section{RESULTS ANDDISCUSSION}

LCM of pure cell populations can allow scientists to obtain specific gene expression signatures from unique cells in their native environment. Retention of RNA quality from these samples will yield the maximum value from quantitative RTPCR or microarray analyses. Proper tissue handling, slide preparation, and sample isolation are essential for obtaining high-quality RNA from LCM samples.

Several researchers have demonstrated that successful isolation of a high-quality RNA sample from LCM material is possible $(4,9,11,14,15)$. However, RNA quality depends critically on time between harvesting and freezing tissue, proper fixation, proper staining, and on choosing an adequate method of RNA extraction and isolation $(4,8,9,14)$. Our HistoGene staining and PicoPure RNA isolation protocols offer
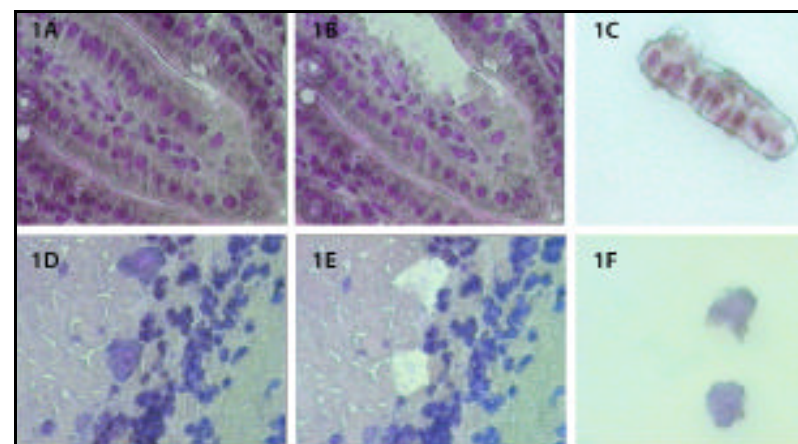

Figure 1. LCM of tissues prepared with HistoGene. Small intestinal villi (A) before and (B) after dissection of the epithelium. (C) Image of the epithelia on the cap. Purkinje cells in cerebellum (D) before and (E) after dissection of the Purkinje cells. (F) Image of the Purkinje cells on the cap. easy, reproducible, reliable, and convenient procedures for tissue slide preparation and sample isolation.

The HistoGene staining procedure is a single-step, rapid procedure that results in good tissue preservation combined with easy and reproducible LCM. Tissue images are superior to those produced using standard hematoxylin \& eosin (H\&E) staining procedures. Identification and microdissection of mouse intestinal epithelium and neurons from mouse cerebellum can be performed with high accuracy after HistoGene staining (Figure 1, A-D). The following cell types/ structures were also efficiently and accurately microdissected from different tissues: hepatocytes from the liver samples, kidney glomeruli, cells residing in the thymic cortex, and secretory units and ducts of the submandibular salivary glands (data not shown).

The PicoPure RNA isolation kit has been developed for optimum extraction, recovery, and preservation of quality RNA from LCM samples. Combined, these two kits allow LCM users to obtain high-quality RNA from their frozen tissues.

The HistoGene staining and the PicoPure RNA isolation process was tested on a variety of different mouse tissues. We demonstrated the isolation of quality RNA through RT-PCR. The message for GAPDH could be easily amplified from 500 cells microdissected from brain, salivary gland, small intestine, and thymus (data not shown). Furthermore, the power of

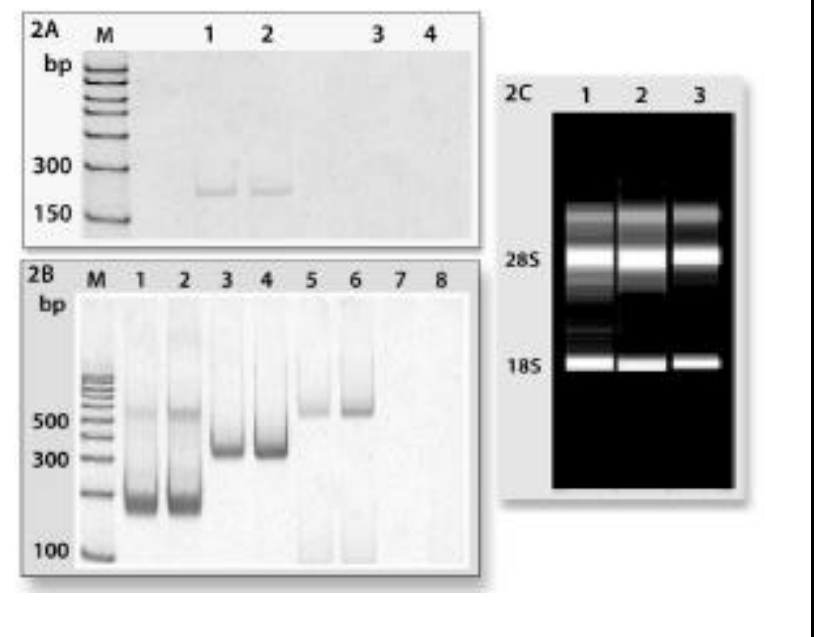

Figure 2. High-quality RNA isolated from LCM samples. (A) RT-PCR detecting $\beta$-actin in RNA isolated from 10 Purkinje cells microdisssected from cerebellum. Lanes 1 and 2, 10 Purkinje cells; lane 3, 10 Purkinje cells, no reverse transcriptase; lane 4, no cDNA. (B) Low-, medium-, and high-abundance genes are detectable in LCM samples prepared with HistoGene. Control RT-PCR primers for genes of varying abundance levels were used on cDNA from 500 cells microdissected from frozen sections of mouse liver and kidney. Lane 1, elongation factor-1 $\alpha$ (high-abundance gene, $187 \mathrm{bp}$ ) detected in kidney; lane 2, elongation factor- $1 \alpha$ in liver; lane 3, GAPDH (mediumabundance gene, $357 \mathrm{bp}$ ) in kidney; lane 4, GAPDH in liver; lane 5, protein phosphatase 1 (low-abundance gene, 498 bp) in kidney; lane 6, protein phosphatase 1 in liver; lane 7, elongation factor- $1 \alpha$ in liver, no reverse transcriptase; lane 8, no cDNA. (C) Electrophoretic analysis of total RNA isolated from LCM samples. RNA was prepared from 15000 cells captured from a section prepared according to the HistoGene protocol (lane 1), an intact frozen section of mouse liver (lane 2), and 15000 cells captured from an unstained but dehydrated section (lane 3) and analyzed on the Bioanalyzer 2000. 
our process was proven by the detection of $\beta$-actin message from as few as 10 Purkinje cells, approximately equivalent to 200 pg total RNA (Figure 2A).

To further evaluate the quality of the RNA obtained from low numbers of cells isolated by LCM from tissues prepared according to the HistoGene protocol and extracted by the PicoPure procedure, we measured the expression of high, medium, and low abundance level genes (Figure 2B). We detected elongation factor- $1 \alpha$ (high abundance), GAPDH (medium abundance), and protein phosphatase 1 (low abundance) message in both liver and kidney microdissections. Our results indicate that even low copy number mRNA species can be isolated from LCM samples and that they appear to be predominantly intact.

The gross integrity of the isolated RNA was determined by microchannel electrophoresis. Sections of kidney were prepared according to our HistoGene staining protocol, extracted with the PicoPure RNA extraction kit, and ribosomal RNAs were analyzed on the Bioanalyzer 2000. We compared unstained and stained material obtained by LCM of 15000 cells from a frozen section of kidney. Figure $2 \mathrm{C}$ shows that the $18 \mathrm{~S}$ and $28 \mathrm{~S}$ bands from the stained section are comparable to those from the unstained section, indicating that there is no deterioration of RNA quality during slide staining and that RNA quality was preserved by our extraction protocol.

RNA from tissue slides prepared by our process was converted to Cy5-labeled cDNA and successfully hybridized to cDNA microarrays that allow the monitoring of expression levels of thousands of genes from LCM samples (data not shown). The use of microarrays and quantitative RT-PCR allows us to survey rapidly and quantitate the expression levels of thousands of genes. To maximize the value of this information, RNA from specific cell populations taken from their native environment should be analyzed. Such samples can now be obtained using HistoGene staining and PicoPure RNA isolation procedures on laser capture microdissected cells.

\section{ACKNOWLEDGMENTS}

This work was partially supported by the Juvenile Diabetes Foundation grant no. 1-2001-56 and the National Institutes of Health grant no. R21 AI 47574.

\section{REFERENCES}

1.Banks, R.E., M.J. Dunn, M.A. Forbes, A. Stanley, D. Pappin, T. Naven, M. Gough, P. Harnden, and P.J. Selby. 1999. The potential use of laser capture microdissection to selectively obtain distinct populations of cells for proteomic analysis-preliminary findings. Electrophoresis 20:689-700

2.Bonner, R.F., M. Emmert-Buck, K. Cole, T. Pohida, R. Chuaqui, S. Goldstein, and L.A. Liotta. 1997. Laser capture microdissection: molecular analysis of tissue. Science 278:1481-1483.

3.Brown, M.R., R. Chuaqui, C.D. Vocke, A. Berchuck, L.P. Middleton, M.R. Emmert-Buck, and E.C. Kohn. 1999. Allelic loss on chromosome arm 8p: analysis of sporadic epithelial ovarian tumors. Gynecol. Oncol. 74:98-102.

4.Dolter, K.E. and J.C. Braman. 2001. Small-sample total RNA purification: laser capture microdissection and cultured cell applications. BioTechniques 30:1358-1361.
5.Emmert-Buck, M.R., R.F. Bonner, P.D. Smith, R.F. Chuaqui, Z. Zhuang, S.R. Goldstein, R.A. Weiss, and L.A. Liotta. 1996. Laser capture microdissection. Science 274:998-1001.

6.Emmert-Buck, M.R., J.W. Gillespie, C.P. Paweletz, D.K. Ornstein, V. Basrur, E. Appella, Q.H. Wang, J. Huang, et al. 2000. An approach to proteomic analysis of human tumors. Mol. Carcinog. 27:158-165.

7.Fend, F., L. Quintanilla-Martinez, S. Kumar, M.W. Beaty, L. Blum, L. Sorbara, E.S. Jaffe, and M. Raffeld. 1999. Composite low grade B-cell lymphomas with two immunophenotypically distinct cell populations are true biclonal lymphomas. A molecular analysis using laser capture microdissection. Am. J. Pathol. 154:1857-1866.

8.Goldsworthy, S.M., P.S. Stockton, C.S. Trempus, J.F. Foley, and R.R. Maronpot. 1999. Effects of fixation on RNA extraction and amplification from laser capture microdissected tissue. Mol. Carcinog. 25:86-91.

9.Kohda, Y., H. Murakami, O.W. Moe, and R.A. Star. 2000. Analysis of segmental renal gene expression by laser capture microdissection. Kidney Int. 57:321-331.

10.Leung, S.M. 2000. A novel microscale approach to disease biomarker discovery from laser capture microdissected cells. Ciphergen Biomarker Discovery Series No. 1.

11.Luo, L., S.C. Salunga, H. Guo, A. Bittner, K.C. Joy, J.E. Galindo, H. Xiao, K.E. Rogers, J.S. Wan, M.R. Jackson, and M.G. Erlander. 1999. Gene expression profiles of laser-captured adjacent neuronal subtypes. Nat. Med. 5:117-122.

12.Lutchman, M., S. Pack, A.C. Kim, A. Azim, M. Emmert-Buck, C. van Huffel, Z. Zhuang, and A.H. Chishti. 1999. Loss of heterozygosity on $8 \mathrm{p}$ in prostate cancer implicates a role for dematin in tumor progression. Cancer Genet. Cytogenet. 115:65-69.

13.Mueller, O., K. Hahnenberger, M. Dittmann, H. Yee, R. Dubrow, R. Nagle, and D. Ilsley. 2000. A microfluidic system for high-speed reproducible DNA sizing and quantitation. Electrophoresis 21:128-134.

14.Ohyama, H., X. Zhang, Y. Kohno, I. Alevizos, M. Posner, D.T. Wong, and R. Todd. 2000. Laser capture microdissection-generated target sam ple for high-density oligonucleotide array hybridization. BioTechniques 29:530-536.

15.Sgroi, D.C., S. Teng, G. Robinson, R. LeVangie, J.R. Hudson, Jr., and A.G. Elkahloun. 1999. In vivo gene expression profile analysis of human breast cancer progression. Cancer Res. 59:5656-5661.

16.Simone, N.L., R.F. Bonner, J.W. Gillespie, M.R. Emmert-Buck, and L.A. Liotta. 1998. Laser-capture microdissection: opening the microscopic frontier to molecular analysis. Trends Genet. 14:272-276.

17.Sirivatanauksorn, Y., V. Sirivatanauksorn, S. Bhattacharya, B.R. Davidson, A.P. Dhillon, A.K. Kakkar, R.C. Williamson, and N.R. Lemoine. 1999. Evolution of genetic abnormalities in hepatocellular carcinomas demonstrated by DNA fingerprinting. J. Pathol. 189:344-350.

18.Tam, A.S., J.F. Foley, T.R. Devereux, R.R. Maronpot, and T.E. Massey. 1999. High frequency and heterogeneous distribution of p53 mutations in alfatoxin B1-induced mouse lung tumors. Cancer Res. 59:36343640 .

19.Thibodeau, S.N., G. Bren, and D. Schaid. 1993. Microsatellite instability in cancer of the proximal colon. Science 260:816-819.

Address correspondence to Dr. Anna Mikulowska-Mennis, Arcturus, 400 Logue Ave., Mountain View, CA 94043, USA. e-mail:amennis@arctur.com 